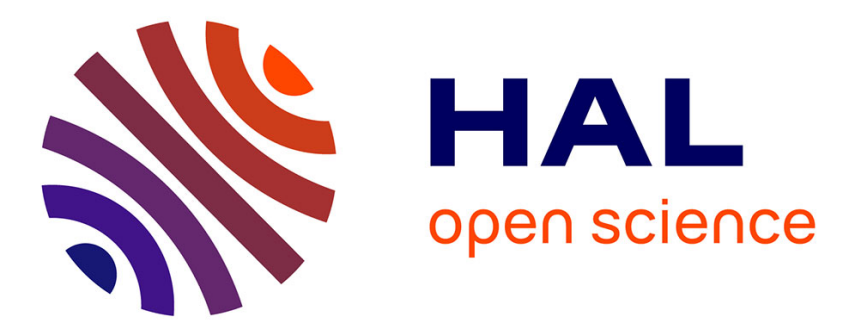

\title{
La construction de l'éthos professionnel en formation alternée
}

Anne Jorro

\section{To cite this version:}

Anne Jorro. La construction de l'éthos professionnel en formation alternée. Travail et apprentissage, 2009, 3, pp.13-25. halshs-00429805

\section{HAL Id: halshs-00429805 https://shs.hal.science/halshs-00429805}

Submitted on 4 Nov 2009

HAL is a multi-disciplinary open access archive for the deposit and dissemination of scientific research documents, whether they are published or not. The documents may come from teaching and research institutions in France or abroad, or from public or private research centers.
L'archive ouverte pluridisciplinaire HAL, est destinée au dépôt et à la diffusion de documents scientifiques de niveau recherche, publiés ou non, émanant des établissements d'enseignement et de recherche français ou étrangers, des laboratoires publics ou privés. 


\title{
La construction de l'éthos professionnel en formation alternée
}

\author{
Anne Jorro \\ Université de Toulouse, CREFI-T
}

Cet article étudie la construction de l'éthos professionnel de stagiaires engagés dans un master professionnel $2^{\text {ème }}$ année «Accompagnement et conseil en développement professionnel ». Issus de la formation initiale et du monde professionnel, ces stagiaires cherchent à construire un positionnement professionnel adapté à l'idée qu'ils se font de la professionnalité du métier visé. La quête de professionnalité exprime un rapport aux valeurs du monde du travail et se traduit par des expressions plus prosaïques «faire pro...être pris au sérieux, devenir un interlocuteur crédible...dépasser le rôle de l'étudiant...». Motivés par le désir de vouloir agir comme un professionnel dans les métiers du conseil, ils font l'expérience de transformations identitaires, ils cherchent à acquérir une culture socio - professionnelle et tentent de construire des répertoires d'action. L'identification des stagiaires à l'image du professionnel attendu suppose l'appropriation de règles, de normes et usages du monde du travail.

Nous disposons de deux corpus de recherche pour approcher cet objet complexe qu'est l'éthos professionnel et tenter de saisir les dimensions axiologiques du développement professionnel dans le cadre de la formation alternée.

Mots clés : éthos professionnel, acte professionnalisant, valeurs professionnelles, reconnaissance professionnelle, formation alternée

\section{Abstract :}

This article studies the construction of the professional ethos of trainees registered in the second year of a professional master course on "Support and advice in professional development".

Coming straight from their studies and from the professional world, these trainees try to build a professional position linked to the idea they have of the professionality in a chosen career. The search for professionalism expresses a connection to the value of the world of business and finds more prosaic expression in such terms as "to be a pro..., to be taken seriously, becoming a credible interlocutor". Identification of the trainees with the professional anticipated assumes appropriation of rules, standards and practices of the business world. Two corpora of research are available to approach the complexity of professional ethos and to try to seize the axiological dimension of professional development in the framework of alternative training.

Keywords :

professional ethos, axiological dimension, professional development, alternative training. 


\section{I- L'éthos professionnel : un objet insaisissable}

Dans le champ du développement professionnel, l'éthos professionnel reste une notion aux contours peu définis alors que le concept d'éthos est particulièrement étudié en linguistique et en pragmatique du langage. La conceptualisation de l'éthos professionnel qui sera ici présentée prend appui sur ces courants de recherche et s'élargit aux approches interactionnelle et axiologique.

Issu de la distinction aristotélicienne logos / pathos / éthos, l'éthos relèverait de l'image de soi dans le discours. Image d'authenticité et d'honnêteté qui donnerait du crédit à l'orateur de sorte que l'interlocuteur serait convaincu par son discours. Dans cette conception, la posture énonciative du locuteur joue un grand rôle : la pragmatique du langage développera l'idée que les conditions d'énonciation importent pour faire valoir une image de soi influente. Les travaux d'Amossy (1999) et de Maingueneau (2002) permettent de saisir la force illocutoire de l'éthos discursif. Le pouvoir du langage donne au locuteur un positionnement identitaire et social permettant une présence discursive. L'éthos organiserait une distribution des rôles entre interlocuteurs et induirait un rapport de places.

Cette théorisation intéresse notre problématique dans la mesure où les stagiaires énoncent un positionnement par rapport à leur développement professionnel, de façon plus ou moins assurée devant le formateur, le référent professionnel, le groupe de formation, les acteurs en situation de travail. L'éthos professionnel s'énoncerait dans des formes langagières marquant alors un degré d'appropriation d'une culture professionnelle donnée.

Une seconde approche retient notre attention, celle de l'interactionnisme symbolique qui constitue une avancée vers la conceptualisation de l'éthos professionnel. Cette approche met l'accent sur l'image de soi dans les interactions sociales (Goffman, 1973). Le langage sert ici un objectif identitaire dans un environnement social donné. La question du rôle tenu par l'acteur dans le contexte social peut être rapprochée de la posture professionnelle mobilisée par le stagiaire en stage. En osant s'afficher comme un futur professionnel, le stagiaire se projette dans une image professionnelle qui l'encourage à agir. L'interaction avec autrui et le désir de se montrer à la hauteur des enjeux professionnels déterminent le stagiaire à aller de l'avant. La «face » serait préservée dès lors que le stagiaire montrerait la pertinence de son action dans l'espace de travail investi.

Enfin, la dimension axiologique intervient dans la conceptualisation de l'éthos professionnel. Il s'agit alors de relever les valeurs qui structurent l'orientation de l'action, qui fondent un type de discours et d'action en relation avec les normes et usages du métier visé. Les critères de beauté et d'efficacité, habituellement mobilisés dans le monde du travail (Clot 1999, Barbier 2001), démontrent l'importance d'une dimension axiologique. Dans le contexte du développement professionnel, l'éthos professionnel met en valeur le positionnement de l'acteur du point de vue de l'image du professionnel attendu. Dès lors, l'éthos professionnel s'apparente à une quête identitaire particulière. La construction identitaire en jeu prend appui sur l'une des deux formes de l'identité théorisée par Ricœur (identité - idem en opposition à l'identité - ipse, 1991), identité pérenne dans laquelle l'acteur peut se reconnaître parce qu'elle renvoie à une façon d'être stabilisée et pour laquelle il devient possible de dire que l'identité - idem répond au genre professionnel du métier visé. Théorisé par la psychologie du travail, le concept de genre professionnel (Clot, 1999) renvoie à la dimension structurante de l'activité et, notamment, aux invariants opératoires. Il relèverait également d'une mémoire impersonnelle et collective, que le stagiaire tente de s'approprier pour agir selon les règles du métier. 
Dans notre recherche, l'éthos professionnel désigne la manière dont le stagiaire organise son rapport au monde professionnel, comment il définit et redéfinit son aire d'action au regard d'une approche déontique de l'activité (Dejours, 1993). L'éthos renvoie donc au principe d'action éthique accompagnant l'agir professionnel. En recherche de mobilisation des savoirs d'action congruents avec «ce qu'il convient de faire», le stagiaire cherche à se positionner selon une approche métier. Il se situe dans un rapport d'introjection en voulant correspondre à ce qu'il peut appréhender du métier d'un point de vue socio - professionnel et ce qu'il est en mesure de s'approprier. Le fait de penser l'action et de s'y projeter relèvent d'un dialogue intérieur souvent difficile à saisir dans les entretiens. Avec l'éthos professionnel, il s'agit de rendre explicite la manière dont «le métier est entré dans l'individu », selon la belle formule de Clot (2008).

La conceptualisation de l'éthos professionnel constitue une première étape de la recherche, une deuxième étape attend le chercheur : l'éthos professionnel est un objet difficile d'accès. Il mobilise le registre de la perception productrice d'impressions dont on interrogera la fiabilité. Il mobilise aussi le registre de l'interprétation comme si cet objet apparaissait en un second temps. Le fait d'attester de l'éthos professionnel exigerait une activité seconde, une lecture en retour. Une approche compréhensive est donc en jeu pour caractériser la construction d'une image du soi professionnel. La difficulté de saisie de l'objet nécessitait une recherche collaborative. En négociant la recherche avec les acteurs (l'échantillon comportait une promotion de 21 stagiaires), le chercheur pouvait s'entretenir avec les stagiaires au cours de leur formation sur l'objet de recherche. Les entretiens de positionnement professionnel conduits en milieu de formation ont permis de comprendre les interprétations proposées par les stagiaires à partir de l'analyse qu'ils portaient sur leurs propres processus de changement.

Sans cette approche collaborative, il aurait été difficile d'étudier un tel objet. Le chercheur souhaitait recueillir une parole vive de sorte que la posture énonciative qui se manifestait lors des entretiens offrait la possibilité de comprendre les nuances du discours. Nous avons procédé à des entretiens de positionnement professionnel que nous avons croisé avec des questionnaires distribués avant ces entretiens : nous avons également recueilli les auto évaluations écrites qui figuraient dans le mémoire professionnel en juin 2008. Cet ensemble de données a fait l'objet d'analyses qualitatives thématiques. En comparant ces deux corpus de recherche, nous avons été en mesure de proposer une première caractérisation de l'éthos professionnel vécu par les stagiaires. Cette première recherche a donc un statut exploratoire et appelle des recherches complémentaires.

\section{II - Du vouloir agir au pouvoir d'agir comme professionnel}

L'éthos professionnel constitue une visée de la formation, chaque stagiaire est sensibilisé à la nécessité de construire une image de soi comme futur professionnel (dans le cas des étudiants) ou comme professionnel différent (pour les professionnels en reconversion). Les démarches de clarification du projet professionnel, d'autoévaluation constituent autant de points d'appui permettant de réfléchir à un positionnement professionnel juste. En outre, l'expérience du groupe de formation et celle des mises en situations professionnelles participent à la construction du positionnement en question. Les discours tenus par les responsables et les formateurs convergent vers la culture du développement professionnel : désormais l'étudiant est sollicité pour réfléchir à ses enjeux d'apprentissage, aux milieux professionnels du conseil 
en développement professionnel. Pour certains stagiaires, le discours est nouveau et particulièrement déstabilisant, pour d'autres le désir d'éthos professionnel s'extériorise par une implication dès le début de la formation, notamment lors des démarches de recherche de stage.

L'engagement dans une dynamique de professionnalisation s'accompagne de prises de consciences successives, parfois coûteuses : nécessité de se connaître pour élaborer un projet, de se dégager des formulations vagues pour cibler des enjeux d'apprentissage. L'objectif de construction de l'ethos professionnel plonge les stagiaires au cœur de tensions identitaires qui les obligent à porter un regard approfondi sur leur positionnement en formation et sur la différence que revêt un positionnement dans le monde professionnel. Cette distinction permet de revisiter ce qui est de l'ordre du développement personnel et ce qui renvoie à un développement professionnel. Le dispositif de formation place les sujets au centre de trois grands processus :

- un processus d'anticipation vers le monde professionnel et par conséquent de projection de soi dans l'univers professionnel,

- $\quad$ un processus de construction identitaire provoqué par la diversité des expériences vécues en stage, à l'université...

un processus d'incorporation des savoirs d'action, des normes et des valeurs propre au genre professionnel visé.

Nous porterons un éclairage sur les deux premiers processus et traiterons du troisième plus loin dans le texte.

\section{Se projeter vers l'éthos professionnel}

La première tâche proposée en formation centre les stagiaires sur la clarification du projet de professionnalisation. Par des retours successifs, les stagiaires précisent les orientations en termes de métiers ou de secteurs visés mais ne sont pas en mesure de formuler des objectifs d'apprentissage, d'identifier les compétences s'y rapportant. La formalisation d'un projet de professionnalisation demande du temps, elle prend une forme explicite lorsque les stagiaires commencent leur stage long d'une durée de deux mois et demi. C'est donc cinq mois après le début de la formation, que la formalisation trouve son aboutissement. Chaque stagiaire a eu le temps de vivre un stage court d'une semaine, de bénéficier d'interventions d'universitaires et de professionnels et de travailler de façon individuelle et collective sur son propre projet professionnel (en entretien individuel en milieu de formation, lors des situations collectives de clinique des pratiques).

Il ressort des entretiens individuels que la difficulté d'une telle formalisation est réelle, unanimement reconnue même si les stagiaires sont motivés. Ce n'est donc pas par absence de motivation que cette difficulté apparaît, elle naît de la connaissance des enjeux professionnels qui les attendent. "J'arrive sur la dernière ligne droite, après je suis sur le marché du travail. Je savais que cette année allait être pour moi déterminante parce que c'est là où je fais le choix, après ça y est je vais être lancée ».

Les tensions identitaires jouent un rôle de filtre dans la formalisation du projet de professionnalisation et tant que l'image de soi est fragilisée par le manque de confiance en soi, il est quasiment impossible d'aller plus loin. Le stagiaire ne peut déterminer finement ses enjeux d'apprentissage, se sentant accaparé par des questionnements identitaires. Se projeter constitue une difficulté d'autant plus grande qu'il a construit tout au long des études universitaires une position d'effacement, un habitus de passivité qu'il lui faut désormais 
abandonner. La difficulté tient dans l'apprivoisement de cette tension identitaire car il s'agit alors de travailler à une identité négociée (Bajoit, 2003, Kadouri, 2006, Wittorki, 2007). Cependant, les difficultés vécues par la plupart des stagiaires n'entament pas le désir d'éthos professionnel tant ils souhaitent construire « une identité pour l'action » et se "noyer dans la masse, ne plus être (des) débutant(s) mais être considérés comme des collègues à part entière et s'adapter à la culture de l'entreprise ». La motivation à "vouloir agir comme un professionnel » suppose un temps intermédiaire où l'horizon professionnel est pressenti sans donner la certitude de l'atteindre. Cette phase de projection vers l'ethos professionnel est exprimée avec un langage marqué par l'ambivalence entre le désir de se confronter à une réalité professionnelle et la difficulté à se doter d'une stratégie de développement professionnel.

Pour les professionnels en reconversion, la situation est différente puisque ces derniers déclarent «savoir ce qu'ils veulent ». Ayant mûri, avant l'entrée en formation, leur projet professionnel, le processus de formalisation du projet de professionnalisation leur apparaît nécessaire et tout à fait réalisable. Cependant, la formalisation oblige à des clarifications qui sont sources de surprises! L'expérience peut constituer un obstacle dans la clarification du projet de professionnalisation.

\section{- Cheminer vers l'éthos professionnel}

Les processus de changement à l'œuvre dans la construction de l'éthos professionnel ne relèvent pas d'un trajet rationnel mais d'un ensemble de détours, de pas de côté qui s'apparentent au cheminement du sujet, cherchant à comprendre ses mutations tout en les vivant. La transformation à l'œuvre s'organise en deux ou trois phases. Une stagiaire identifie trois temps: "un temps dans une posture d'étudiante en recherche de stage, un temps dans une posture d'étudiante stagiaire au moment de la signature de la convention de stage, un temps dans une posture de future professionnelle dans la réalisation des missions et de rencontres avec les professionnels ».

Nous soulignerons deux mutations identitaires vécues comme essentielles :

- De l'attitude d'étudiant(e) à la posture de stagiaire : les stagiaires les plus motivés font la différence entre l'attitude qu'ils pouvaient avoir à l'université dans les formations classiques et l'attitude qu'ils gagnent dans un parcours de professionnalisation. A la passivité de l'étudiant, ces stagiaires très engagés dans la formation répondent par l'envie d'en découdre et de se débrouiller. Bourgeois (2006) souligne que l'implication du sujet en formation serait d'autant plus forte que le stagiaire saisirait la valeur ajoutée de celle-ci dans la poursuite de sa quête identitaire.

Sur une promotion de 20 étudiants, un quart est resté dans un « rôle d'étudiant classique » tout au long du parcours de formation, prenant conscience en fin de cursus de la nécessité de se positionner autrement sans vraiment être en mesure de vivre cette mutation identitaire. Le manque de confiance en soi, le fait de ne pas oser se lancer ont généré une forme d'immobilisme. Les stagiaires qui sont entrés dans une dynamique identitaire expriment souvent l'obstacle psycho-affectif qui les inhibe un temps et qu'ils ont combattu à leur manière. Cette recherche est confortée par les travaux de Chaix (2008).

- Une stagiaire revient sur les manières de se présenter devant autrui et évoque son changement vestimentaire :

" avant je m'habillais très souvent en jean, j'avais des baskets, des tee shirt un peu rigolos, un peu à l'arrache : bon j'avais un piercing et cette année franchement avec le stage on a bien vu les milieux dans lesquels on allait être plongés et les postures qu'on 
allait devoir tenir, les rôles qu'on allait devoir jouer et tout ça m'a permis de grandir un peu plus... c'est lié le développement professionnel avec le développement personnel donc, par exemple, au niveau vestimentaire je m'efforce quand j'achète un pantalon, ce n'est plus un jean, c'est un pantalon en toile voilà ».

- De la posture de stagiaire à celle de «presque collègue » : ce passage naît avec le regard d'autrui. Il concerne les stagiaires qui ont pu en stage mettre en œuvre des actions qu'ils présentent comme enrichissantes et qui peuvent apporter un service au collectif de travail.

Cette position nouvelle suppose un effort certain, celui d'oser faire face, d'oser dialoguer dans la difficulté, d'oser parler de l'importance de travailler sur des missions "grandeur nature», ou sur des missions qui situent le stagiaire au coeur d'apprentissages spécifiques. A ce prix, le regard des professionnels peut changer et le stagiaire agir comme novice. "Au début du stage, je me sentais dans la peau d'un stagiaire. Au fur et à mesure que le temps a passé, j'ai senti que l'on me faisait plus confiance et que ma part d'autonomie devenait plus large. Ma posture de stagiaire devenait une posture de collègue avec tout de même une certaine réserve».

L'analyse qui est produite ici tend à souligner l'importance des contextes et notamment la tension que vit le stagiaire pour construire une «posture professionnelle négociée » en fonction des paramètres de la situation. Les professionnels du terrain ne sont pas toujours prêts à porter dès le début du stage un regard approbateur sur le stagiaire et c'est le degré de résistance et de négociation du stagiaire, par rapport à la mission qui lui est confiée et aux tâches qui en dépendent, qui le positionne comme interlocuteur devant le référent professionnel ou le personnel.

Les mutations identitaires en jeu sur le terrain du stage permettent alors d'évoluer du rôle imparti de stagiaire à la posture professionnelle négociée. Autant le rôle est quasiment défini à l'avance et impose un type de comportement, autant la posture consiste en une identité agie, adaptée au contexte. Ce mouvement du rôle vers la posture professionnelle négociée constitue un apprentissage coûteux pour le stagiaire mais valorisant dans la mesure où il a su négocier sa place dans un espace socioprofessionnel organisé. Le passage du rôle de stagiaire à celui de presque collègue exige parfois des précautions. Les stagiaires s'engagent trop vite dans l'activité alors que les acteurs de terrain apprécieraient une présence moins marquée sur leur territoire. Quelques situations de stage ont été un lieu d'apprentissage du positionnement professionnel à tenir devant autrui de sorte que la présence ne soit pas vécue par les salariés comme une intrusion dans leur espace de travail. La construction de l'éthos professionnel appelle non seulement une prise de conscience sur le vouloir agir comme professionnel mais aussi sur les transactions professionnelles au travail. Se faire admettre dans un espace de travail appelle aussi un positionnement professionnel.

Nous avons fait la différence pour les métiers de l'éducation et de la formation entre le fait de vouloir jouer un rôle et d'agir une posture (Jorro, 2002). Dans le cas des stagiaires, on observe à la fois, l'envie de jouer un rôle en voulant agir parfois trop vite, provoquant ainsi des remous sur le lieu de stage, et la possibilité de construire une posture acceptable par tous. Cette mutation identitaire ne peut opérer que lorsque les transactions professionnelles sont positives. Cette situation de développement professionnel est particulièrement éclairante parce qu'elle met en évidence l'importance des contextes de travail, parfois difficiles d'accès, résistants à tout changement, offrant alors des cadres d'expériences au stagiaire (Goffman, 1991). 
Pour les professionnels en reconversion, la problématique est différente. Le statut de professionnel en reconversion les situe d'emblée en voie de perfectionnement dans un autre contexte. La crédibilité est déjà acquise, aussi c'est la valeur ajoutée du stage pour la structure d'accueil qui sera appréciée. Les enjeux identitaires sont d'une autre teneur, il ne s'agit pas de se lancer dans la réalité d'un contexte professionnel mais de construire ses marques dans un espace de travail afin de confirmer un projet de reconversion et d'incorporer de nouvelles compétences professionnelles. Toutefois, les professionnels reviennent systématiquement sur la nécessité de se former théoriquement, d'analyser leurs pratiques. Le fait «d'avoir des bases » mais aussi le désir de développer une posture réflexive l'emportent sur le fait de se voir agir ailleurs et faire autre chose ou bien de se voir agir dans la même structure avec une autre mission.

\section{III - L'incorporation des valeurs professionnelles}

L'éthos professionnel constitue un positionnement souhaité dans le contexte de travail. Il agrège un certain nombre de valeurs qui relèvent de la professionnalité d'un acteur. Les qualités professionnelles, sur lesquelles nous ne reviendrons pas, ont été décrites ailleurs (Le Boterf, 2007) sur lesquelles nous ne reviendrons pas. Il reste que l'analyse des entretiens et des questionnaires permet de noter les interactions profondes entre les valeurs personnelles et professionnelles dans la construction du positionnement professionnel. On peut avancer l'idée que, par la conscientisation de ces valeurs, le stagiaire entre dans un processus d'identification à un genre professionnel. Le cheminement vers l'éthos professionnel dépendrait :

- d'une part, de la découverte des valeurs professionnelles qui permettent alors une identification à une identité de métier. Les métiers du conseil sont porteurs de valeurs humanistes auxquelles adhèrent largement les stagiaires. De façon unanime, les stagiaires énoncent les valeurs d'écoute, de respect de la personne, de dialogue...qui sont centrales dans les métiers du conseil et notamment dans la relation aux différents publics. L'identification au métier commence par le contact avec les gens de métier qui sont remarqués pour leur savoir faire mais plus encore pour les valeurs « qui transpirent » de leurs gestes professionnels. Dans certains cas, les rencontres et les dialogues constituent des moments d'identification. Lorsque la relation s'établit, les professionnels sont observés et imités. Une stagiaire déclare vouloir connaître les dimensions informelles d'un service pour agir au mieux. Il y a une volonté d'acculturation pour entrer dans l'espace de travail et connaître les règles du jeu. Le rôle des enseignements dans la dimension axiologique reste faible, le dit reste secondaire au regard de l'expérience de l'action. L'immersion en contexte, la rencontre des acteurs constituent des marqueurs axiologiques.

- d'autre part, de la prise de conscience de l'intériorisation de ces valeurs. Lorsque les valeurs professionnelles s'expriment dans l'action et qu'elles sont identifiées, le stagiaire se trouve conforté dans son activité, il entrevoit la pertinence du positionnement professionnel adopté. La prise de conscience de l'intériorisation des valeurs professionnelles consolide son évolution identitaire. Autrement dit, les valeurs agies façonnent un habitus professionnel et confirmeraient le stagiaire dans son « désir de métier »(Osty, 2003).

- mais aussi, de la légitimation par le terrain de la mobilisation pertinente de ces valeurs. A partir d'un retour réflexif avec le référent professionnel, les valeurs identifiées, le réglage de l'action participent à la construction du positionnement professionnel du stagiaire. Ainsi une stagiaire, dit qu'elle appréhende mieux la posture de conseiller après une situation de coévaluation avec le référent au cours de laquelle son implication a été analysée et évaluée. 
Cette validation déboucherait sur une forme de reconnaissance du positionnement professionnel tenu. Le processus de légitimation serait dépendant de la qualité de l'interaction professionnelle. Bien des situations d'interaction entre un référent professionnel et un stagiaire ne débouchent pas sur une légitimation du positionnement professionnel (Trohel \& alii, 2004).

-Et encore de la promotion par l'université de la valeur de réflexivité

La construction de l'éthos professionnel dépend également de l'expérience que le stagiaire s'est forgée dans l'alternance formation - mises en situations professionnelles. Les valeurs de réflexivité qui caractérisent le professionnel les conduisent à vouloir prendre de la distance avec les situations épineuses ou à structurer leur démarche pour mieux agir. Ces valeurs professionnelles sont énoncées comme fondatrices du rapport que les stagiaires tissent avec l'idée du positionnement d'un professionnel. Ces valeurs de réflexivité sont associées à la dimension éthique. En cherchant à saisir le champ d'action qui est le leur ou en repérant les limites de leur action, les stagiaires ajustent leur positionnement professionnel. Ce qui est la marque d'une intériorisation de ce qui peut et doit être fait.

\section{IV - L'incorporation des savoirs d'action à travers les actes professionnalisants}

La construction de l'éthos professionnel est étroitement liée à la mise en œuvre d'une activité dans le contexte professionnel, laquelle constitue un saut qualitatif vers la professionnalisation. Le processus de professionnalisation est majoré lorsque le stagiaire vit une expérience professionnelle en grandeur réelle, qu'il a le sentiment d'avoir participé à la dynamique de travail d'un service ou d'une entreprise et que, par la mission qui lui a été confiée, il a su gagner une autonomie nouvelle. Le développement professionnel opère alors à partir de la co-transformation acteur-action- terrain (Jorro, 2007).

L'analyse des entretiens nous conduit à revenir sur la relation étroite entre le pouvoir d'agir (Ricoeur, 2004) du stagiaire et la construction de l'éthos professionnel. Ce pouvoir d'agir se manifeste à travers des actes professionnalisants. Ces actes marquent une différence avec la situation initiale telle qu'elle se présentait avant l'intervention du stagiaire. L'acte professionnalisant revêt le caractère d'un passage à l'acte qui transformera le stagiaire, lui donnant le sentiment d'avoir gagné une étape dans son processus de professionnalisation. Autrement dit, l'activité effective crée un écart significatif qui situe le stagiaire à une autre étape de son développement professionnel. L'acte professionnalisant, vécu comme une expérience significative, relève alors du sentiment de réussite (Bourgeois, 2006). Si l'acte professionnalisant est vécu comme un acte réussi par celui qui le commet, c'est qu'il est en mesure de faire la différence avec un savoir détenu, une démarche antérieure. A la suite des travaux de Van Gennep (1987), nous pourrions dire que l'acte professionnalisant est constitutif du passage de différents seuils. Avant de faire, au moment d'agir, et après l'action représentent ces trois seuils :

- avant de faire, le stagiaire puise dans ses ressources personnelles et environnementales pour définir une démarche qui lui paraît pertinente ;

- au moment d'agir, il se donne la motivation nécessaire pour se lancer dans l'activité, il ose agir ;

- après l'action, il peut analyser la qualité de son action, capitaliser ce savoir, et le rendre disponible pour d'autres situations.

Le stagiaire se destinant vers le monde professionnel abandonne son parcours antérieur pour se consacrer pleinement à l'épreuve de l'activité dont il pourra tirer sa propre lecture critique 
et se construire une ligne de conduite. En vivant les trois phases spécifiques des rites de passage, les stagiaires comprennent le poids de l'expérience vécue en stage. Les mises en situations professionnelles les transforment à la condition qu'elles aient fait l'objet de situations réflexives.

Pour l'ensemble des stagiaires, les actes professionnalisants sont marqués au moins par quatre traits :

1. L'activité porte la marque du monde professionnel et s'inscrit dans une organisation avec des objectifs, des coutumes, des normes...Elle se joue en grandeur réelle, elle est authentique. Elle provoque des modifications chez le sujet, notamment sur les stagiaires qui vivent une première expérience. Pour les professionnels en reconversion cette dimension importe mais sur un autre plan car il s'agit pour eux de changer de contexte professionnel.

2. L'expérience de l'activité professionnelle a une valeur inédite au sens où le stagiaire a effectué une action nouvelle pour lui, a eu le sentiment de sortir de lui-même, de se découvrir dans une situation nouvelle. Il a éprouvé une manière d'être et d'agir ; en ce sens il a vécu une expérience cruciale (Vygotsky, 1997).

3. L'activité constitue une épreuve personnelle pour le stagiaire qui a vécu une situation inconfortable parce qu'incertaine quant au résultat attendu et encore imprévisible dans son déroulement. Cette dimension est évoquée de façon quasi immédiate par les stagiaires en formation initiale, elle apparaît dans le discours des professionnels en reconversion dans un second temps.

4. L'activité fait l'objet d'un retour réflexif : l'acte ne devient professionnalisant que pour autant qu'il fasse l'objet d'une analyse et d'une évaluation de son bien fondé, de sa pertinence et de son efficacité. Ce retour réflexif est possible avec les démarches accompagnées (Bourassa \& alii 1999, Boutinet 2007, Paul, 2004).

\footnotetext{
Le passage à l'acte :

Le fait de rédiger un $C V$ pour décrocher un stage.

La construction d'outils : outils de coordination d'équipe, référentiels de formation, outils d'évaluation, tableaux de bords...

La pratique accompagnée :

"Lors des réalisations des entretiens en binôme je me suis sentie dans mon élément, ce contact, cette écoute, cet accompagnement global et personnalisé, ce respect ainsi que cette équité dans l'accueil et le suivi, correspondent tout à fait aux valeurs qui sont les miennes. Ce constat m'a conforté dans mon choix d'orientation professionnelle vers le secteur social, ainsi que celui du conseil et par conséquent vers la profession de conseiller en insertion professionnelle ou socioprofessionnelle auprès des publics en difficulté. »

Le retour des professionnels :

"En discutant avec le responsable des ressources humaines avec qui je pouvais facilement parler du stage, nous avons pu réfléchir et faire le point sur mon intervention. Le stage globalement s'est bien passé. Mais, il a mis le doigt sur un élément qui m'a remise en question professionnellement et personnellement. J'ai peut - être pris la mission de stage trop à cour en m'impliquant personnellement. Je n'ai pas réussi à mettre de la distance dans mon
} 
intervention. Je me suis trop engagée personnellement car la classe ouvrière me tient relativement à cœur et j'ai souhaité à travers ce stage les aider à améliorer leur situation... ».

Pour une autre stagiaire, le retour du référent professionnel est de l'ordre de la reconnaissance " Je te trouve mois éparpillée, plus professionnelle... »

Pour les professionnels en reconversion, les actes professionnalisants relèvent de l'appropriation et de la mobilisation d'une compétence, notamment de la compétence réflexive, dans plusieurs contextes. Pour ces professionnels, l'acte professionnalisant est donc constitutif du raffinement de la compétence: le professionnel prend conscience des différentes manières de mobiliser une compétence et de son caractère modulaire.

Les effets déclarés de l'acte professionnalisant sur le stagiaire sont de l'ordre de l'accomplissement professionnel parce qu'ils permettent :

- une confiance en soi : l'épreuve de l'activité renforce le positionnement du stagiaire ;

- la confirmation d'une orientation professionnelle : le pouvoir d'agir constitue une autorisation à aller de l'avant, à envisager son futur professionnel ;

- la découverte d'une autonomie dans l'action : le stagiaire découvre l'action dans sa dimension générique et singulière, il apprend à jouer avec l'imprévu, à se confronter à des organisations et aux cultures organisationnelles qui configurent des relations professionnelles ;

- une certaine crédibilité devant autrui : le sentiment de devenir un interlocuteur à part entière du monde professionnel commence avec le regard du référent professionnel et des acteurs en situation de travail.

\section{V- La reconnaissance professionnelle comme horizon d'attente}

Le désir d'être reconnu comme un professionnel à part entière motive les stagiaires quel que soit leur niveau d'expérience. Si les projets sont singuliers, la quête de professionnalité reste un objectif commun. Nous avons vu précédemment que la construction de l'ethos professionnel nécessitait la clarification du projet de professionnalisation, permettant de vivre des mutations identitaires et de vivre l'expérience formatrice des mises en situations professionnelles. Le cheminement $\mathrm{du}$ «vouloir agir au pouvoir d'agir comme un professionnel » trouvant son aboutissement avec le retour réflexif sur l'action. Un retour gratifiant résiderait dans la reconnaissance d'une professionnalité (Jorro, 2009). Et lorsque la reconnaissance professionnelle a lieu, elle émerge de différents contextes :

-tout d'abord, en formation universitaire : elle débute avec le regard des pairs : les stagiaires font la différence des professionnels qui ont de l'expérience et des stagiaires qui cherchent à en acquérir. Ils repèrent un champ de compétence chez autrui, discutent de ce champ de compétences, observent les manières de faire, s'imprègnent de certains gestes.

- puis, sur le terrain de stage à travers les actes professionnalisants et les interactions avec le milieu de travail, la reconnaissance de soi par soi va de pair avec la reconnaissance par autrui. - enfin, la reconnaissance de leur cheminement professionnel trouve son aboutissement lors de la soutenance du mémoire professionnel puisque la validation qui s'ensuit offre une légitimité nouvelle. La présence du référent professionnel en soutenance donne une dimension socio professionnelle à l'appréciation qui est portée.

Une triple reconnaissance émerge donc et nous renvoie aux dimensions que nous dégagions à propos des formes de reconnaissance professionnelle dans la construction des portfolios professionnels (Jorro, 2006). 


\section{Conclusion}

La construction de l'éthos professionnel en formation alternée est tributaire de l'anticipation de soi vers le monde professionnel visé. Cette construction suppose des mutations identitaires et des processus d'incorporation de normes propres au genre professionnel du conseil.

La quête de professionnalité telle qu'elle est exprimée par les stagiaires met en évidence la coconstruction entre identité et valeurs. Avec la coexistence de ces processus de changement, la transformation de l'image de soi opère et un soi professionnel émerge en relation avec le métier visé. Les aspects identitaires et axiologiques «infiltrent» les dimensions opératoires de l'activité.

Cette recherche exploratoire ne nous permet pas encore de caractériser finement la spécificité de l'éthos professionnel selon le rapport que les acteurs entretiennent avec l'expérience professionnelle. Une difficulté majeure attend le chercheur, celle qui concerne la sémantique de l'activité. Les stagiaires n'ont pas les mots pour dire le désir de métier qui les mobilise. A leur manière, ils expriment l'intériorisation active de l'identité à partir du moment où ils cherchent à «être du métier». De même, la construction de l'éthos à partir des transactions propres au monde du travail et propres au groupe de formation reste à élucider. Si l'éthos professionnel constitue un levier parmi d'autres du développement professionnel des stagiaires, la question du désir de métier nous paraît de première importance.

\section{Bibliographie}

Amossy, R. (1999). Images de soi dans le discours. Lausanne : Delachaux et Niestlé.

Bajoit, G. (2003). Le changement social. Paris : Armand Colin.

Barbier, J.-M. (2001). Quelques questions pour la recherche dans le domaine de l'évaluation, dans G. Figari et M. Achouche (éd.), L’activité évaluative réinterrogée. Paris, De Boeck, p. 351-360.

Bourassa, B., Serre, F. \& Ross, D. (1999). Apprendre de son expérience. Québec : PUQ.

Bourgeois, E. (2006). Tensions identitaires et engagement en formation. In J-M. Barbier, E. Bourgeois, G. de Villers \& M. Kaddouri (eds) Constructions identitaires et mobilisation des sujets en formation. P. 65-120. Paris : L'Harmattan.

Boutinet, J-P. (2007). Penser l'accompagnement adulte. Paris : PUF.

Chaix, M-L. (2008). Chaix, M-L. (2008). Co-construction de savoirs et d'identités dans les dispositifs alternants. Communication au $15^{\text {ème }}$ congrès de l'AMSE, Mondialisation et éducation, Marrakech.

Clot, Y. (1999). La fonction psychologique du travail. Paris : PUF.

Clot,Y. (2008). Travail et pouvoir d'agir. Paris : PUF.

Dejours, C. (1993). Travail. Usure mentale. Paris : Bayard.

Goffman, E. (1973). La présentation de soi. Paris : Minuit.

Goffman, E. (1991). Les cadres de l'expérience. Paris : Minuit.

Jorro, A. (2009). La reconnaissance professionnelle : évaluer, valoriser, légitimer. Ottawa : PUO.

Jorro, A. (2007). Evaluation et développement professionnel. Paris : L'Harmattan.

Jorro, A. (2006) Les formes de la reconnaissance professionnelle dans la démarche de portfolio in Figari, G. Rodriguez, P., Alves, P. , Valois, P. (eds). Evaluation des compétences et apprentissages expérientiels: savoirs, modèles et méthodes. p.143-151. Lisbonne: EDUCA.

Jorro, A. (2002). Professionnaliser le métier d'enseignant. Paris : ESF. 
Kaddouri, M. (2001). Vers une typologie des dynamiques identitaires. In Centre de recherches sur la formation (ed). Action et identité. P.163-175. Paris : INRP.

Le Boterf, G. (2007) Professionnaliser. Paris : EO.

Maingueneau, D. (2002). Problèmes d'éthos. Pratiques, n¹13/114, p. 57-68.

Osty, F. (2003). Le désir de métier. Rennes : Presses universitaires de Rennes.

Paul, M. (2004). L'accompagnement : une posture professionnelle spécifique. Paris : L' Harmattan.

Ricoeur, P. (2004). Parcours de la reconnaissance. Paris : Seuil.

Ricoeur, P. (1991). Soi-même comme un autre. Paris : Seuil.

Trohel, J., Chalies, S., Saury, J. (2004). La dynamique des interactions tuteurs-stagiaires en situation de conseil pédagogique. Savoirs, 5, p.121-140.

Van Genep, A. (1987). Les rites de passage. Etude systématique des rites. Paris: Aet J. Picard.

Vygotsky, L.V. (1997). Pensée et langage. Paris : La Dispute.

Wittorski, R. (2007). Professionnalisation et développement professionnel. Paris :

L'harmattan 